\title{
Absence of evidence of superconductivity in sulfur hydride in optical reflectance experiments
}

\author{
J. E. Hirsch ( $\nabla$ jhirsch@ucsd.edu ) \\ University of California San Diego \\ F. Marsiglio \\ University of Alberta
}

\section{Short Report}

Keywords: optical reflectance, energy gap, superconductivity, electron-phonon interaction

Posted Date: October 14th, 2021

DOI: https://doi.org/10.21203/rs.3.rs-967287/v1

License: (9) This work is licensed under a Creative Commons Attribution 4.0 International License. Read Full License 


\title{
Absence of evidence of superconductivity in sulfur hydride in optical reflectance experiments
}

\author{
J. E. Hirsch ${ }^{a}$ and F. Marsiglio ${ }^{b}$ \\ ${ }^{a}$ Department of Physics, University of California, San Diego, La Jolla, CA 92093-0319 \\ ${ }^{b}$ Department of Physics, University of Alberta, Edmonton, Alberta, Canada T6G 2E1
}

PACS numbers:

Capitani and coworkers [1] reported that infrared optical reflectance measurements provided evidence for a superconducting transition in sulfur hydride [2] under $150 \mathrm{GPa}$ pressure, and that the transition is driven by the electron-phonon interaction. Here we argue that the measured data did not provide evidence that the system undergoes a transition to a superconducting state, nor do the data support any role of phonons in driving a transition. Rather, the data are consistent with the system remaining in the normal state down to temperature $50 \mathrm{~K}$, the lowest temperature measured in the experiment. This calls into further question $[3,4]$ the generally accepted view [5] that sulfur hydride under pressure is a high temperature superconductor.

We requested all the raw data used in Ref. [1] from the corresponding authors, and obtained them for temperatures $50 \mathrm{~K}, 100 \mathrm{~K}, 150 \mathrm{~K}, 240 \mathrm{~K}$ and $320 \mathrm{~K}$. The paper also shows data for temperatures $200 \mathrm{~K}, 130 \mathrm{~K}$ and 170 $\mathrm{K}$; we reiterated our request for those raw data but did not receive them.

Figure 1 shows raw data for $\mathrm{H}_{3} \mathrm{~S}$ (main frame). This data represents an intensity detected at the spectrometer, and is comprised of a combination of blackbody source radiation and reflectance off the sample and diamond anvil apparatus [6]. Also shown in the bottom right inset for comparison is the (presumably raw) reflectance data from a cuprate sample [7]. In an attempt to remove non-intrinsic signals from the $\mathrm{H}_{3} \mathrm{~S}$ data, the authors of Ref. [1] plotted ratios of intensity at different temperatures. This is shown in the inset to the immediate right of the main figure, while the corresponding procedure for the cuprates is given at the far right side (top).

It is clear that at low temperature, the cuprate reflectance flattens and approaches unity on the scale given by the superconducting energy gap $2 \Delta_{0}$, estimated as $2 \Delta_{0} \approx 350 \mathrm{~cm}^{-1}=43.4 \mathrm{meV}$ for that case. All cuprate reflectance curves, below and above $T_{c}$, monotonically increase with decreasing frequency/energy (except for some sharp dips due to phonons) and approach unity at low frequency/energy, and for a given frequency, the reflectance monotonically decreases as the temperature increases. In the inset showing the ratio of the reflectance for the two lowest temperatures for the cuprate (far right at the top), a peak is present, consistent with the expectation given in Fig. 1b of [1]. One of these curves is at a temperature above $T_{c}$, and also shows a gap, consistent with the original authors' interpretation of their normal state data in

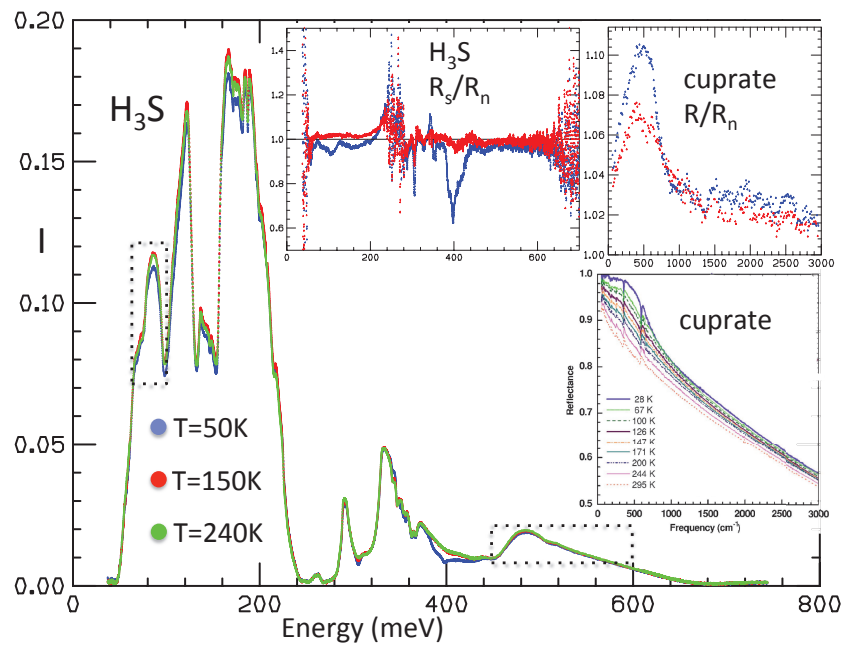

FIG. 1: Raw data for measured reflectance of sulfur hydride underlying the results presented in Ref. [1]. The dotted rectangles mark the regions of energy shown in Figs. $2 \mathrm{~b}$ and $3 \mathrm{~b}$ of Ref. [1]. The lower right inset shows reflectance data for a cuprate superconductor, with $T_{c}=59 \mathrm{~K}$ from Ref. [7]. To convert frequency $\left(\mathrm{cm}^{-1}\right)$ to $\mathrm{meV}$, multiply by 0.124 . The upper left and right insets show reflectance ratios for $\mathrm{H}_{3} \mathrm{~S}$ and for the cuprate respectively: $R_{n}$ is for $T=240 K(T=200 K)$ for the left (right) upper inset; $R_{s}$ in the upper left inset is for temperature $T=50 K$ (blue) and $T=150 K$ (red). $R$ for the upper right inset is for $T=28 K$ (blue) and $T=67 K$ (red).

terms of a pseudogap. The contrast with the complicated pattern in the inset on the left showing reflectance ratios for $\mathrm{H}_{3} \mathrm{~S}$ is striking.

The usual technique of evaporating gold on a freestanding sample to obtain an absolute reflectance is not feasible here [6]. It is clear that the main intensity in the body of Fig. 1 has large contributions from sources other than the $\mathrm{H}_{3} \mathrm{~S}$ sample. Using Eq. (3) in the Supplementary Material of Ref. [1] we can relate the ratio of the sample reflectance in the superconducting and normal state to the ratio of intensities at the same two temperatures:

$$
\frac{R_{s}}{R_{n}}=\frac{I_{s}}{I_{n}}-\frac{a}{R_{n}}\left(1-\frac{I_{s}}{I_{n}}\right),
$$

where the parameter $a=0.13$ if we use the values for $R_{c}$ and $\alpha$ provided in Ref. [1]. Since the second term on the right-hand-side of Eq. (1) is at most of order $2 \%$ and tends to correct the ratio towards unity, we approximate the ratio of reflectances by the ratio of intensities. 

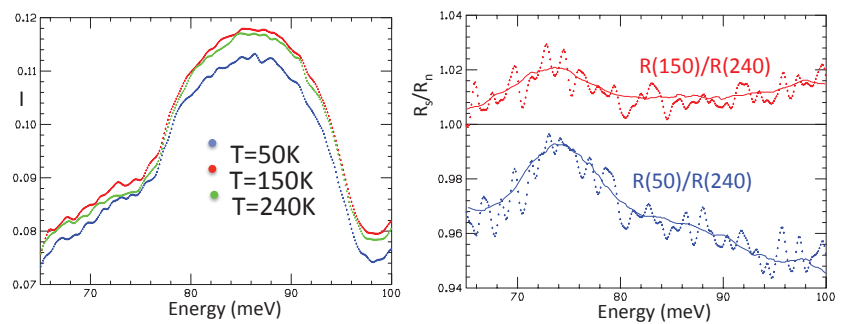

FIG. 2: Left panel: intensity raw data. Right panel: reflectance ratios. The lines show the curves displayed in Fig. $2 \mathrm{~b}$ of ref. [1], shifted down (up) by 0.042 (0.016) for the blue (red) curve.

It is clear from comparison of the upper left and right insets that the reflectance ratios in the case of $\mathrm{H}_{3} \mathrm{~S}$ do not indicate superconductivity.

To zoom in on this issue, in Fig. 2 we show the intensity raw data and ratios in the energy range $65 \mathrm{meV}-100$ meV that Ref. [1] focuses on to infer that $\mathrm{H}_{3} \mathrm{~S}$ undergoes a superconducting transition. The solid curves on the right panel are taken from Fig. 2b of [1]; however, to match the measured data we had to shift the blue curve given in Ref. [1] downward by 0.042 and the red curve upward by 0.016 . Note that these are significant shifts on the scale shown. The fact that the low temperature reflectance ratio is smaller than the high temperature reflectance ratio, opposite to what Ref. [1] shows, directly contradicts the interpretation that these features are "in good agreement with the theoretical gap structure" as is claimed in Ref. [1]. The left panel of Fig. 2 shows that both the temperature and energy dependence of the measured curves are dominated by effects not coming from the sample of interest, $\mathrm{H}_{3} \mathrm{~S}$.

Ref. [1] also shows reflectance ratios in their Fig. 3b in the energy range $450 \mathrm{meV}$ to $600 \mathrm{meV}$, reproduced here on the right panel of Fig. 3, and they claim that their positive slope and temperature dependence is in agreement with theoretical calculations and "demonstrates that $\mathrm{H}_{3} \mathrm{~S}$ is an Eliashberg superconductor driven by the electronphonon interaction with strong coupling to high phonons of order of $200 \mathrm{meV}$ '. For comparison, on the left panel of Fig. 3 we plot the indicated reflectance ratios obtained from the raw data that we obtained. The red points on the left panel should be the same as the light blue curve on the right panel, but they are not. The blue points on the left panel, for a lower temperature, should have a steeper positive slope as the dark blue curve on the right panel, but they do not. Instead, both sets of points on the left panel show a rather noisy and flat behavior qualitatively different from the behavior in the right panel in the same energy range, $450 \mathrm{meV}$ to $600 \mathrm{meV}$. For energies below $450 \mathrm{meV}$, not shown on the right panel, the low temperature (blue) curve on the left panel shows a sudden sharp drop which is not predicted by the theory used in Ref. [1] (see the dashed blue line in Fig. 3a of [1]). It has been suggested that it is due to ice depositing on the diamond surface at low temperature [6]. We argue
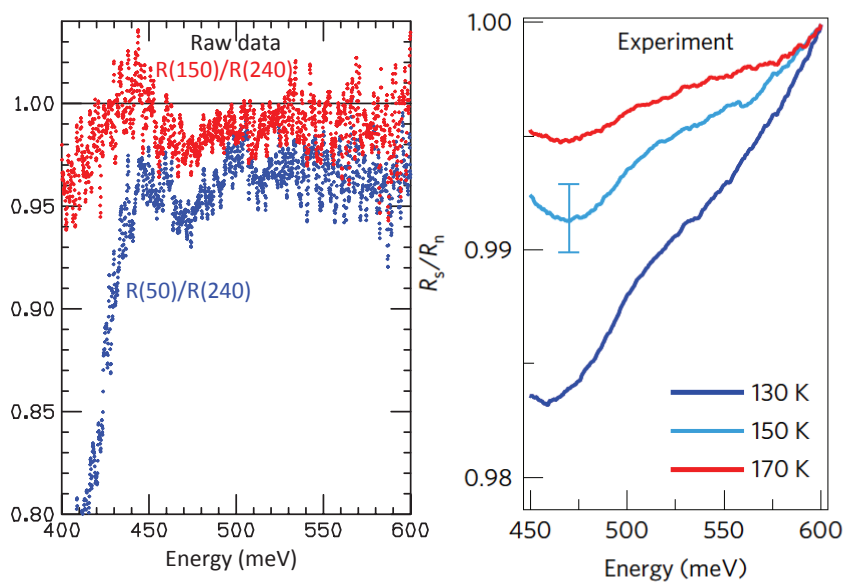

FIG. 3: Left panel: reflectance ratios from raw data. Right panel: curves from Fig. 3b of Ref. [1].

that the measured data shown in the left panel of Fig. 3, which markedly differ from the curves shown in the right panel, provide no support to the hypothesis that Ref. [1] claims the right panel of Fig. 3 "demonstrates".

Returning to Fig. 2b in Ref. [1], the figure caption reads "The blue and red solid curves indicate the measured $R_{s}(T) / R_{n}$ for $T=50 \mathrm{~K}$ and $150 \mathrm{~K}$, respectively with $R_{n}$ measured at $200 \mathrm{~K}$." Given that the energy dependence of the curves in Ref. [1] follows closely the behavior we obtain from the raw data, as shown in our Fig. 2 right panel, we conclude that the paper has a typo and $R_{n}$ was measured at $240 \mathrm{~K}$. However, it is clear from our Fig. 2 that the curves shown in the paper were not "the measured $R_{s}(T) / R_{n}$ ", but rather the measured $R_{s}(T) / R_{n}$ shifted as indicated in the figure caption of our Fig. 2. This shift is crucial since it led to a temperature dependence claimed in the paper exactly opposite to what the measured data showed. The authors concluded from their Fig. 2b that "The intensity of this feature and its temperature dependence are in good agreement with the theoretical behaviour of the superconducting gap". Instead, we conclude that the data plotted in Fig. $2 \mathrm{~b}$ of Ref. [1], rather than being "the measured $R_{s}(T) / R_{n}$ " that the figure caption stated, resulted from alteration of the measured data in unexplained and unjustified ways, and that the measured data provide no support for the existence of a superconducting gap.

In summary, we argue that Ref. [1] presents a misleading picture. Data for carefully selected small energy windows were chosen, ratios of measured quantities rather than absolute values were plotted, and measured data were altered in unexplained ways, to arrive at the published data, in order to hide the complicated and unexpected behavior of the measured data shown in Fig. 1. The purpose of these selections and alterations was clearly to provide apparent support to the a priori assumption that $\mathrm{H}_{3} \mathrm{~S}$ is a high temperature conventional superconductor. As a result, the reader of Ref. [1] is left with the impression that these optical measurements pro- 
vided (i) confirmation that sulfur hydride is a superconductor and (ii) confirmation that the electron-phonon interaction drives its superconductivity. Instead, we have shown here that in fact the measured data provided support for neither. The reality is that the measured data have major contributions from unknown sources that render them incapable of supplying any information about the physics of $\mathrm{H}_{3} \mathrm{~S}$.

\section{Acknowledgments}

The authors are grateful to Tom Timusk and Pascale Roy for providing the raw data and additional informa- tion and for helpful discussions. FM was supported in part by the Natural Sciences and Engineering Research Council of Canada (NSERC) and by an MIF from the Province of Alberta.

Competing interests: there is NO Competing Interest

[1] F. Capitani et al, "Spectroscopic evidence of a new energy scale for superconductivity in $H_{3} S$ ", Nat Phys. 13, 859 (2017).

[2] A.P. Drozdov, M.I. Eremets, I. A.Troyan, V. Ksenofontov and S. I. Shylin, "Conventional superconductivity at 203 kelvin at high pressures in the sulfur hydride system", Nature 525, 73-76 (2015).

[3] J. E. Hirsch and F. Marsiglio, Phys. Rev. B 103, 134505 (2021); Physica C 587, 1353896 (2021); Physica C 584, 1353866 (2021); Physica C 589, 1353916 (2021); Nature 596, E9, (2021).
[4] J. E. Hirsch, arXiv:2109.08517 (2021).

[5] Ref. [2] has over 1,600 citations in Google Scholar as of September 15, 2021.

[6] T. Timusk, private communication; P. Roy, private communication.

[7] J. Hwang et al, Phys. Rev. B 73, 014508 (2006).

[8] E. J. Nicol and J. P. Carbotte, "Comparison of pressurized sulfur hydride with conventional superconductors", Phys. Rev. B 91, 220507(R) (2015). 
Figures

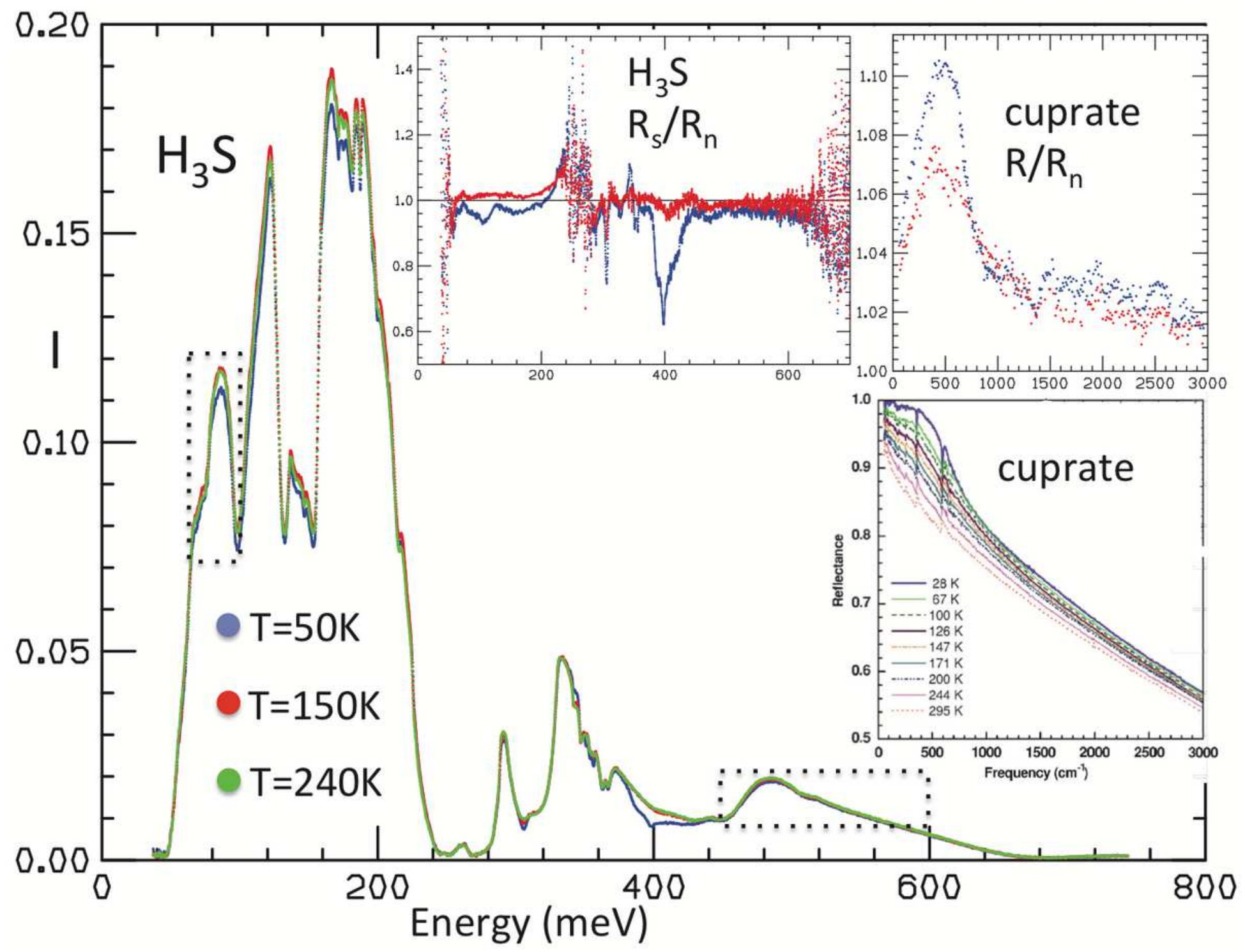

Figure 1

Raw data for measured reflectance of sulfur hydride underlying the results presented in Ref. [1]. The dotted rectangles mark the regions of energy shown in Figs. $2 b$ and $3 b$ of Ref. [1]. The lower right inset shows reflectance data for a cuprate superconductor, with Tc $=59 \mathrm{~K}$ from Ref. [7]. To convert frequency $(\mathrm{cm}-1)$ to meV, multiply by 0.124 . The upper left and right insets show reflectance ratios for H3S and for the cuprate respectively: $\mathrm{Rn}$ is for $\mathrm{T}=240 \mathrm{~K}(\mathrm{~T}=200 \mathrm{~K}$ ) for the left (right) upper inset; $\mathrm{Rs}$ in the upper left inset is for temperature $T=50 \mathrm{~K}$ (blue) and $T=150 \mathrm{~K}$ (red). $\mathrm{R}$ for the upper right inset is for $\mathrm{T}=28 \mathrm{~K}$ (blue) and $\mathrm{T}=67 \mathrm{~K}(\mathrm{red})$. 

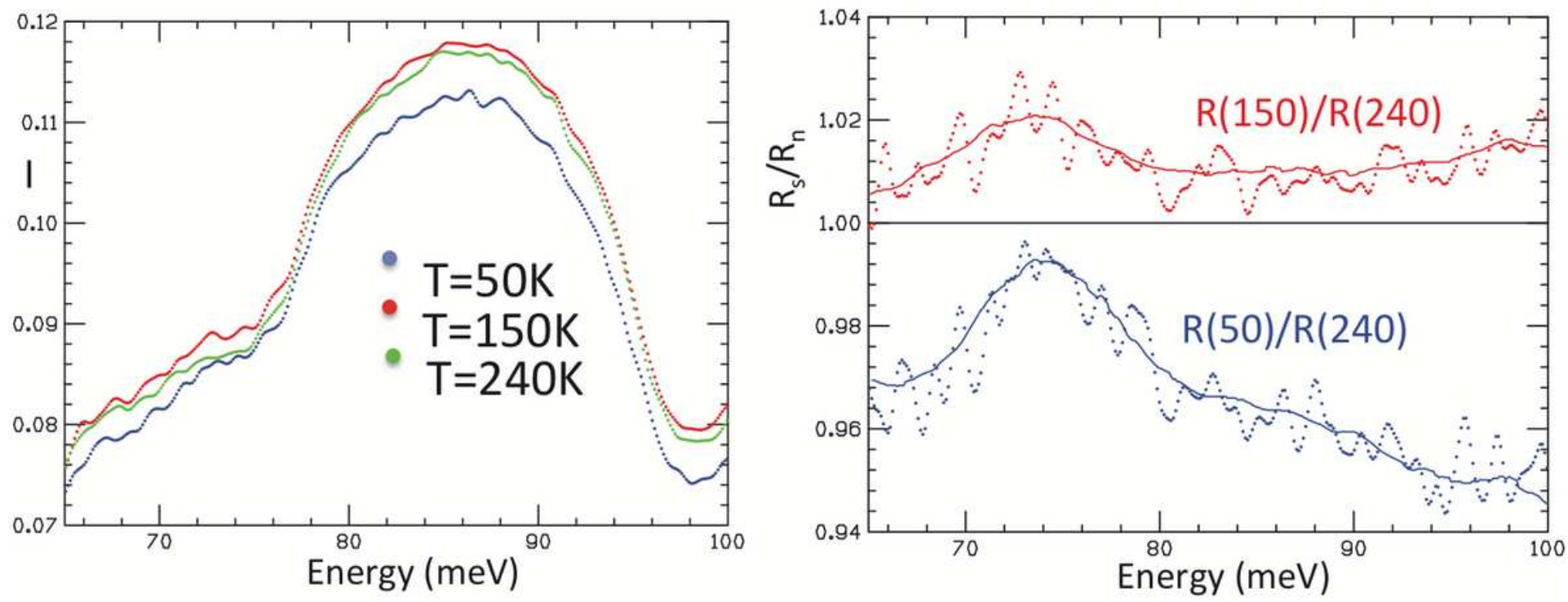

Figure 2

Left panel: intensity raw data. Right panel: reflectance ratios. The lines show the curves displayed in Fig. $2 b$ of ref. [1], shifted down (up) by 0.042 (0.016) for the blue (red) curve.
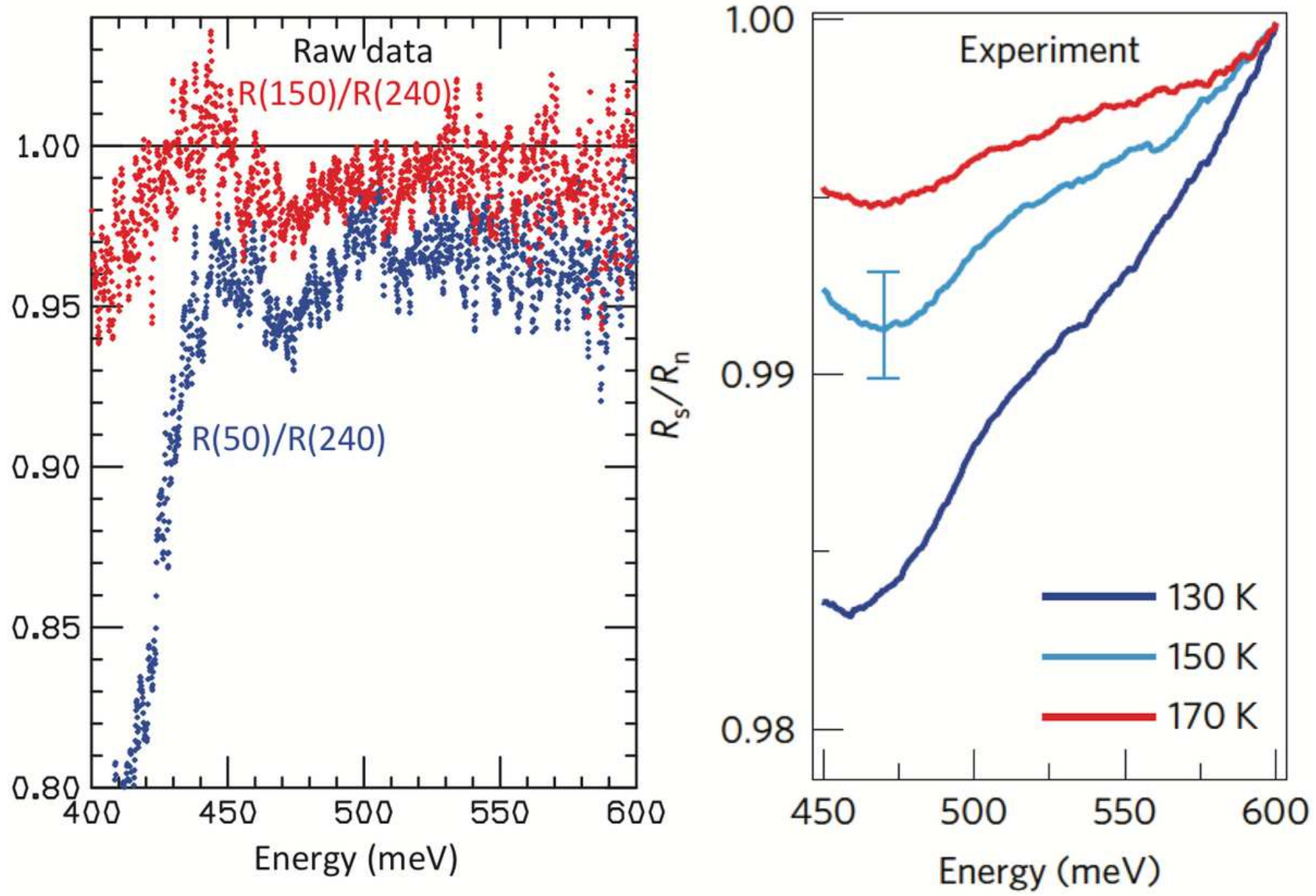

Figure 3 
Left panel: reflectance ratios from raw data. Right panel: curves from Fig. 3b of Ref. [1]. 